\title{
THE STUDY OF CURVES OF THE TRANSIENT CURRENT IN POLYTETRAFLUOROETHYLENE BY MEANS OF FOURIER ANALYSIS
}

\author{
Turdybekov K. M. ${ }^{1}$, Turdybekov M.K. ${ }^{2}$ \\ ${ }^{1}$ E. A. Buketov Karaganda University, Karaganda, Kazakhstan, kalkantur@mail.ru \\ ${ }^{2}$ Karaganda Technical University, Karaganda, Kazakhstan
}

\begin{abstract}
Experimental transition current curves for polytetrafluoroethylene after exposure to high-energy ionizing radiation are obtained. The transient current curves in irradiated polytetrafluoroethylene are analyzed based on the discrete Fourier transform. It is shown that there is an intense maximum of dielectric absorption in the infralow frequency region. This maximum is characterized by abnormally large values of the dielectric increment, permittivity, and the dielectric loss factor. It is concluded that the volume polarization of the irradiated polytetrafluoroethylene is uniform. The polarization observed in this frequency range is associated with the activation of migration of trapped charge carriers within microscopic regions.
\end{abstract}

Keywords: transient current curves, electrophysical properties, polytetrafluoroethylene, ionizing irradiation, Fourier analysis.

\section{Introduction}

The study of the effect of ionizing radiation on the electrical properties of solid dielectrics has become urgent due to the development of modern radiation technologies, the widespread use of insulating materials in nuclear and space research. Polymer materials used in spacecraft as part of screen-vacuum thermal insulation, electrical insulation of cables, thermal control coatings are exposed to the entire energy spectrum of ionizing radiation [1-3]. As an object of study, polytetrafluoroethylene (PTFE) is interesting on the one hand because it is a polymer of high practical significance, due to its unique complex of physics-chemical properties (chemical and biological resistance, excellent dielectric, antifriction and anti-adhesion properties, elasticity in the field of cryogenic temperatures). On the other hand, the fact that it reveals a number of electrical phenomena that have not yet found their explanation and are the subject of discussion [4-10].

The electrophysical properties of polytetrafluoroethylene (PTFE) after ionizing irradiation in the range of absorbed doses of $10^{3}-10^{5}$ Gy degrees have a number of characteristic features. The values of the specific volume conductivity $\left(\sigma_{\gamma}\right)$ of PTFE at room temperature after $\gamma$ - and electron irradiation increase by two or more orders of magnitude depending on the irradiation conditions and the physical state of the object under study (disks, film, thermal Modification) [1]. In the dielectric relaxation spectra of irradiated PTFE, intense maxima appear in the frequency range $10^{2}-10^{4} \mathrm{~Hz}$ at $\mathrm{T}=400-500 \mathrm{~K}$ (after irradiation in vacuum) and $\mathrm{T}=360-400 \mathrm{~K}$ (after irradiation in air) [1]. Similar changes were observed for other fluorinated polymers, as well as for weak and nonpolar polymers [1] and were attributed to the type of slowly reversible changes, since they are characterized by annealing effects. For fluorinated polymers, both reversible and slowly reversible changes in the dielectric characteristics were associated with the accumulation of peroxide macroradicals. However, the estimation of the values of their effective dipole moment under the condition of the maximum possible concentration leads to unreasonable values $\left(\mu_{\mathrm{ef}} \sim 10^{-28} \mathrm{C} \cdot \mathrm{m}\right)$.

For the dipole-group and segmental mobility of most polymers at glass transition temperature $\mathrm{Tgt}<$ $400 \mathrm{~K}$, the specified time interval is sufficient to establish equilibrium polarization. However, after irradiation of polymers, a complete analysis of the transition current curves is necessary to determine the $\sigma_{\mathrm{V}}$ of the polymer matrix, since the relaxation times of the captured charges can be any, or, in any case, this time interval is not known in advance. To this end, this paper studies the transient current curves in the charging and discharging modes of irradiated PTFE in the frequency range: $10^{-4}-10^{-1} \mathrm{~Hz}$. 


\section{Samples and Research Methods}

The study of the transition current of the technical film of polytetrafluoroethylene F4-IO with a thickness of 80 microns was carried out. Metal electrodes (measuring, high-voltage, and guard ring) were applied to the sample by thermal sputtering of silver in a vacuum. The transient current curves were recorded according to standard methods using measuring cells with a small parasitic capacitance, electrometric amplifiers (B7-30, U5-11, BK2-16). The time constant of the current measurements did not exceed $1 \mathrm{~s}$, the $\mathrm{RC}$ - measured circuit did not exceed $10 \mathrm{~s}$. The irradiation was carried out by gamma-rays with an energy of $1.2 \mathrm{MeV}$ in air at the $\mathrm{K}-120$ facility with the isotope $60 \mathrm{Co}$, the absorbed dose $\mathrm{D}=5 \mathrm{Mrad}$.

Transition current curves of a technical PTFE film with a thickness of 80 microns were studied. The analysis of transient current curves before and after gamma irradiation was performed using a block of standard Fourier analysis programs and original blocks of input-output programs and processing of Fourier analysis data implemented on a computer. Experimental points for calculations were taken after $1 \mathrm{~s}$ in the range $0-100 \mathrm{~s}$, after $10 \mathrm{~s}$ in the range $100-1000 \mathrm{~s}$, and after $100 \mathrm{~s}$ in the range $1000-10000 \mathrm{~s}$.

The transition current curves after switching on (charging current) and off (discharging current) of the voltage source for the original PTFE are straightened in double logarithmic coordinates. The exponent of the time dependence of the discharge transient current density $j(t) \sim t^{-n}$ for the initial PTFE is $\eta=1.6$ (Fig. 1). This result can be interpreted in terms of the Debye model for dipole-group mobility. If there is a Cole-Cole distribution of dipole relaxation times at $\mathrm{t}>\tau$ (the dipole relaxation times are obviously much less than the characteristic measurement time) $(t / \tau)^{\alpha-2}$, [11], where $\tau$ is the relaxation time of the dipoles, and is the parameter of the relaxation time distribution. Based on the experimental dependences, we obtain the value $\alpha=$ 0.4 , which is typical for dipole-group losses.

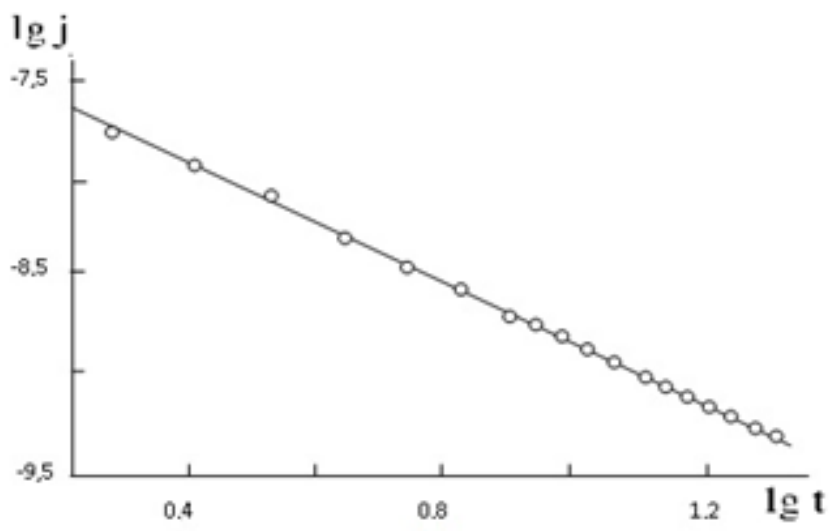

Fig.1. Kinetics of the discharge current density of an unirradiated PTFE film.

For the initial PTFE samples, the transient current density usually reached values less than $10^{-11} \mathrm{~A} / \mathrm{m}^{2}$ by the first minute, which corresponds to $\sigma_{\mathrm{v}}<10^{-17} \mathrm{Om}^{-1} \cdot \mathrm{m}^{-1}$ and is consistent with the literature data[11]. The dielectric increment can be determined from the discharge current curves:

$$
\Delta \varepsilon=\int_{0}^{\infty} j(t) d t / \varepsilon_{0} E_{0} \cong 2 \cdot 10^{-2}
$$

where $\varepsilon_{0}$ is the dielectric constant, $\mathrm{E}_{0}$ is the polarizing field strength, and $\mathrm{t}$ is the time in seconds.

The concentration of dipoles at normal values of $\mu_{e f} \sim 10^{-30} \mathrm{C} \cdot \mathrm{m}$ will be

$$
N=3 \varepsilon_{0} k T \cdot \Delta \varepsilon / \mu^{2} \cong 10^{25} \mathrm{~m}^{-3}
$$

and corresponds to the concentration of chemical and physical defects in PTFE. The occurrence of a polarizing current due to ionogenic impurities at room temperature is unlikely. These effects are usually observed at elevated temperatures. A completely different situation is observed for irradiated PTFE. The values of the transient current density in the initial section of the kinetic curve are three or more orders of magnitude higher than the corresponding values for the initial samples, and the current drop is observed up 
to $10^{4} \mathrm{~s}$ (Fig.2). Moreover, the discharge current of depolarization differs little in absolute value from the charging current.

The estimate of $\Delta \varepsilon$ by gives values for various PTFE films irradiated in air in the range of absorbed doses of $10^{4}-10^{5} \mathrm{~Gy}$, from 30 to 80 . Obviously, such high values of $\Delta \varepsilon$ cannot be provided by dipole polarization. Even if their local concentration is high and the volume distribution is inhomogeneous, the recorded values of transient currents would need to be further increased, since the value of the integral in (1) is averaged over the volume.

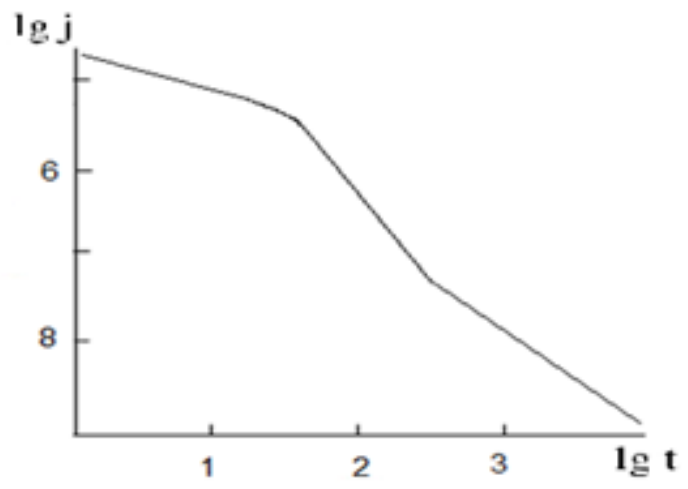

Fig.2. Kinetics of the discharge current density of a $\gamma$-irradiated PTFE film up to $\mathrm{D}=50 \mathrm{kGy}$ in air.

It follows from the above that the transition current curves up to $10^{4} \mathrm{~s}$ are due to the establishment of polarization due to microscopic displacement of charges. In this regard, the increase in $\sigma_{\mathrm{v}}$ recorded after PTFE irradiation is mainly due to polarization currents, rather than stationary conductivity [1].

\section{Discussion of results}

Charge-discharge curves when recorded repeatedly on the same sample are well reproduced at room and low temperatures. Minor annealing effects begin to appear at $310 \mathrm{~K}$ and almost complete annealing, when the discharge curves differ slightly from the corresponding curves for the original samples, was observed after recording the charging curve for $10^{4} \mathrm{~s}$ at $470 \mathrm{~K}$. To determine the nature of polarization, we compared the discharge currents in two modes. In the first mode, the circuit during charging and discharging is shorted according to Fig.3a, in the second mode, the polarity of switching on during discharge changes to the opposite (Fig.3b). When an excess charge density is formed at one of the electrodes, the discharge currents in these switching schemes should differ markedly, just as it is observed when the excess charge injected into the surface layers of the dielectric is relaxed. The discharge curves observed by us were almost identical in both switching schemes. Thus, the polarization of irradiated PTFE can be characterized as uniform volumetric.

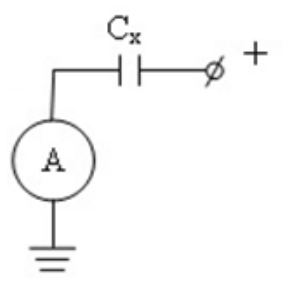

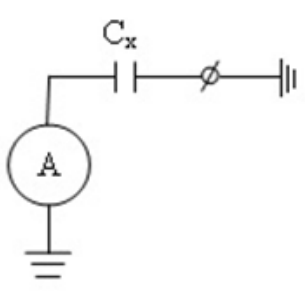

a

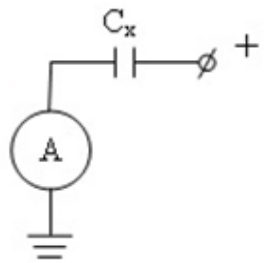

b

Fig.3. Scheme for measuring discharge currents without changing (a) and with a variable (b) polarity of switching on samples.

It is known that the time dependence of the transient current and the corresponding frequency dependence of the conductivity are related by the Fourier transform $[11,12]$. In the presence of an exponential response $\mathrm{j}(\mathrm{t}) \sim \exp (-\mathrm{t} / \tau)$, the variance of the transient current density is not observed, since the relative changes in the exponential function are the same at any time. For $j(t) \sim t-s$, the relative changes in the transient current density decrease with increasing time, which corresponds to an increase in conductivity with increasing frequency. Experimental results indicate the existence of a frequency dependence of the conductivity of irradiated PTFE. Moreover, the presence of inflection points on the charge-discharge curves 
clearly indicates the non-monotonic nature of this relationship. We have analyzed transient currents based on the discrete Fourier transform. It is known $[11,12]$ that

$$
\left.\varepsilon^{\prime}(\omega)-i \varepsilon^{\prime \prime}(\omega)=\int_{0}^{\infty} j(t)\right) \exp (-i \omega t) d t / \varepsilon_{0} E_{0},
$$

then

$$
\begin{aligned}
& \varepsilon^{\prime}\left(\omega_{i}\right)=\int_{0}^{\infty} j(t) \cos \omega_{i} t d t / \varepsilon_{0} E_{0}, \\
& \varepsilon^{\prime \prime}\left(\omega_{i}\right)=\int_{0}^{\infty} j(t) \sin \omega_{i} t d t / \varepsilon_{0} E_{0},
\end{aligned}
$$

where $\omega_{i}=2 \pi / t_{i}$

The dispersion curves $\varepsilon^{\prime}$ and $\varepsilon^{\prime \prime}$ obtained from the Fourier analysis of transient currents correspond to the infra-low frequency region $10^{-4}-10^{-1} \mathrm{~Hz}$. As can be seen in Fig. 4, the maximum of dielectric absorption is formed in the specified frequency range. The character of the curves $\varepsilon^{\prime}$ and $\varepsilon^{\prime \prime}$ fully corresponds to the Debye relations for the real and imaginary parts of the permittivity [12-14]. Moreover, the values of $\varepsilon^{\prime}, \varepsilon^{\prime \prime}$ in the maximum and $\Delta \varepsilon$ are an order of magnitude or more higher than the possible values for dipole polarization processes. Lowering the temperature in full accordance with the Debye theory leads to a shift in the maximum towards lower frequencies. The activation energy found from the displacement of the peaks in Arrhenius coordinates was $84 \mathrm{~kJ} / \mathrm{mol}$. The values of the pre-exponential factor $v_{0}=6 \cdot 10^{13} \mathrm{~Hz}$ significantly exceed the corresponding values for the dipole group $\left(v_{0} \cong 10^{13} \mathrm{~Hz}\right)$ and segmental $\left(v_{0} \cong 10^{12} \mathrm{~Hz}\right)$ mobility in polymers, which in turn is consistent with the activation mechanism of migration of captured charge carriers.

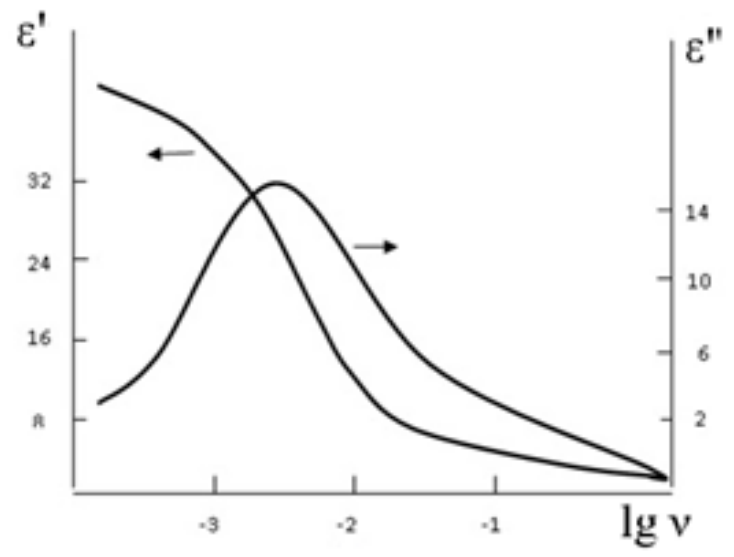

Fig.4. Frequency dependence of the permittivity $\varepsilon^{\prime}$ and the dielectric loss factor $\varepsilon^{\prime \prime}$ for PTFE after gamma irradiation in air; $\mathrm{D}=50 \mathrm{kGy}$.

Thus, the analysis of transient currents in the frequency range $10^{-4}-10^{-1} \mathrm{~Hz}$ shows that the polarization effects observed for irradiated PTFE can be interpreted by thermal activation of charge carriers both with the participation (near $400 \mathrm{~K}$ ) and without the participation (at $\mathrm{T}<300 \mathrm{~K}$ ) of the polymer matrix and their displacement within microscopic regions not related to conductivity. However, it remains unclear the question about the nature of these areas. It could be assumed that the charges are mainly localized on the chemical and physical defects of the structure. However, thermal modification of the samples does not lead to qualitative changes in the spectra, and the corresponding electrophysical parameters remain anomalous. In addition, similar patterns were observed on other fluorinated polymers (and even polyolefins) with significantly different nature of structural traps.

The latter fact clearly indicates the more General nature of the observed polarization phenomena. It can be assumed that the observed effects are related to the inhomogeneous spatial distribution of the ionization regions. These regions can be spurs, blobs, and short tracks formed during the interaction of secondary electrons with matter. The lifetimes of these micro-regions may be quite large, since the dispersion transport 
of charge carriers in them occurs under conditions of strong scattering to deep radiation traps, while the times of dielectric relaxation of the polymer matrix are $\tau=10^{6}-10^{8} \mathrm{~s}$.

\section{Conclusion}

In general, changes in the dielectric parameters are anomalous and are observed both directly during the exposure to radiation, and after its termination for a long time. Several different explanations for the radiation-induced effects of the electric polarization of polytetrafluoroethylene have been put forward in the literature, but a single view on this issue has not yet been developed.

In this paper, we study the volume electric polarization in polytetrafluoroethylene (- CF2-CF2 -) n exposed to high-energy ionizing radiation. The small difference in the absolute value of the charge-discharge currents suggests that there is no significant influence of the near-electrode polarization on the observed process. Since the polarization curves in both inclusion schemes practically did not differ, the polarization of the irradiated polytetrafluoroethylene can be characterized as uniform volumetric. The curves of the polarization and depolarization currents are reproduced repeatedly with an error not exceeding $10 \%$.

A Fourier analysis of the time dependences of the polarization current is performed. The main feature of the obtained results is the abnormally high values of $\varepsilon^{\prime}$ and $\varepsilon^{\prime \prime}$ in the region of the relaxation maximum, which are not typical for elementary dipoles. The analysis shows the existence of an intense maximum of dielectric absorption in the region of infra-low frequencies with abnormally large values of the dielectric increment, permittivity, and the dielectric loss factor. Polarization phenomena in the infra-low frequency region are associated with activation of migration of trapped charge carriers within microscopic regions. These areas are not connected by conductivity. Such areas can be spurs, blobs, and short tracks formed during the interaction of secondary electrons with matter.

\section{REFERENCES}

1. Milinchuk V. K. Radiation resistance of organic materials. Moscow, Energoatomizdat, 1986, 171p. [in Russian].

2. Khatipov S.A. Radiation electrical conductivity of polymers. Chapter 1.12 of the collection «Model of space: The impact of the space environment on the materials and equipment of spacecraft». Moscow, 2007, pp. 361 - 376. [in Russian].

3. Khatipov S.A., Tsvelev V.M., Alekseev S.V. Radiation resistance of polymers in the conditions of outer space. Chapter 1.15 of the collection «Model of space: The impact of the space environment on the materials and equipment of spacecraft». Moscow, 2007, pp. 414-436. [in Russian].

4. Obvintsev A.Yu. and others. The effect of gamma radiation on the surface and bulk properties of polytetrafluoroethylene. The surface. X-ray, synchrotron, and neutron studies. 2017, No. 9, pp. 52-60. [in Russian]

5. Zhutaeva Yu.R., Khatipov S.A. Maxwell-Wagner polarizationin polytetraflyoroethylene sudjected to the impact of ionizing radiation. Proc. of the Intern. Simposium «Physics and Chemistry of processes, oriented toward development of new high technologies, materials and equipment». Chernogolovka, 2007, pp. 317-322.

6. Tyutnev A. P., Arkhipov V. I., Nikitenko V. R., Sadovnichy D. N. On the nature of non-Langevin recombination of charge carriers in polymers. Chemical physics. 1996, vol. 15, No. 3, pp. 91-99. [in Russian].

7. Malyshkina I.A., Burmistrov S.E., Gavrilova N.D. Dielectric spectroscopy of sulfonated polytetrafluoroethylene in the swollen state. High-molecular compounds. 2005, B, Vol.47, No. 8, pp. 1563-1568. [in Russian].

8. Klein R.J., Cole S.M., Belcher M.E., et al. Radiation tolerance in polymeric dielectrics by small molecule doping. Part II: Thermodynamic and kinetic parameters. POLYMER, 2008, Vol. 49, p.5549-5563.

9. Khatipov S. A., Turdybekov K. M., Edrisov A. T., Milinchuk V. K. Electrification of polymer films under the influence of vacuum ultraviolet light. High-energy chemistry. 1995, Vol. 29, No.3, pp. 184-186. [in Russian]. Russian].

10. Sergeeva A. E. Polarization and spatial charge in ferroelectric polymers. Odessa: TES, 2014 , 347 p. [in

11. Lushcheikin G. A. Methods for studying the electrical properties of polymers. Moscow: Khimiya, 1988,160 p. [in Russian].

12. Sazhin B. I. Electrical properties of polymers. Leningrad, Chemistry, 1986, 224 p. [in Russian].

13. Lushcheikin G. A. Polymer electretes. Moscow, 2008, 180 p. [in Russian].

14. Sessler G. M. Electrets. Springer-Verlag, Berlin. 2003, 437 p. 